\title{
Diagnostic framework for attachment disorders needs improving
}

Reactive and Disinhibited Attachment Disorders (RAD and DAD) occur when infants and young children have not been able to form an attachment to their primary caregiver, but questions have been raised as to whether these disorders are being over-diagnosed in adopted children. Matt Woolgar and Emma Baldock performed a case review of 100 attendees of a National Child and Adolescent Mental Health Service for adopted children.

They found that attachment disorders were four times over-represented in referral letters compared to the number of actual diagnoses made in the clinic. This over-diagnosis at referral may be due to inadequate diagnostic criteria and use of inappropriate terminology and poor symptomatic support at referral. Over-referral for attachment disorders did not obscure the diagnosis of other disorders. In fact, diagnosed cases of conduct disorders or ADHD in the clinic were worryingly under-represented in referral letters by up to 10-fold. The researchers conclude that attachment disorders should be specified as RAD or DAD at initial referral and that the current framework for diagnosing RAD/DAD needs improvements.

Woolgar, M. \& Baldock, E. (2015), Attachment disorders versus more common problems in looked after and adopted children: comparing community and expert assessments. Child Adolesc Ment Health, 58:770-778. doi:10.1111/camh.12052 